\title{
Relation Between the Porosity of Wool Assembly and its Tendency of Sinking in Liquids
}

\author{
By Haruo Sanuki, Member, TMSJ \\ Tokyo University of Agriculture and Technology, Koganei, Tokyo
}

\begin{abstract}
To analyze the fundamental behavior of a wool assembly in steeping and washing bowls, the author has studied the process in which an assembly floating on a liquid surface begins to sink. The time $\left(t_{B}\right)$ needed for an assembly to begin sinking has been measured for various values of porosity $\left(P_{h}\right)$ by using distilled water of various temperatures, trichlene and methyl alcohol of a normal temperature and a solution of non-ionic wetting agent of various concentrations $\left(C_{p}\right)$ and of a normal temperature.

The difference in $t_{s}$ between various values of $P_{h}$ is small if the concentration of the wetting agent is high (dense). The difference in $t_{s}$, however, is high for a low concentration of the wetting agent, the maximum of $t_{h}$ occuring at $P_{h}=80 \%$. The relation between $\log t_{x}$ and $\log C_{p}$ is practically linear and had a constant tangent if the porosity degree is fixed at a standard vlaue. When this wetting agent solution is used, the porosity arranged in order from fastest to slowest sinking of scoured wool is $P_{\Omega}=97>95>90>70>75>85>80 \%$.
\end{abstract}

\section{Introduction}

In two previous articles $(1,2)$ the author analyzed the effects of the degree of porosity (degree of opening) in wool assemblies on the efficiency of their steeping and scouring. The analysis showed that the efficiency of treatment varied greatly with the degree of porosity.

It seems that the treating efficiency in these processes is greatly affected by the degree of penetration of a wool assembly by the treating liquid. Accordingly, to analyze the fundamental behavior of a wool assembly in the steeping and washing bowls, the author thinks it important to compare and study the relation between the degree of permeation and the varying degrees of porosity.

In this article, the author has investigated, as the first step, the process in which a wool assembly floating on a liquid surface begins to sink. The method hitherto used for osmosis tests of wetting agents has been the Draves-Clarkson method or the yarn bundle method. $\{3,4,5 j$ These methods measure and compare the time needed for a yarn hank or a piece of felt to begin sinking. In the author's ex. periment under review, however, he studied how far the sinking of a floating solid varied with its apparent density when a given liquid was used.

\section{Preliminary Measurement}

A preliminary measurement of the time needed for a wool assembly to begin sinking has been measured for various values of porosity and by us. ing distilled water of various temperatures and trichlene and methyl alcohol of a normal temperature. Wool assemblies with different degrees of porosity were prepared by the method of porosity $P_{h}$ in horizontal extension. (6) The method was to make, out of wire netting, cylindrical vessels of a desired capacity and stuff equal quantities of wool fibers $-7.0 \mathrm{~g}$ in absolute dry weight-uniformly into the vessels. Each sample vessel was made equal in total weight, $7.2 \mathrm{~g}$., by attaching a sinker of the proper size to it. The quantities of the liquids used for all measurements were $800 \mathrm{cc}$ each and the active surface area of the liquids $86 \mathrm{~cm}^{3}$.

Tables $1,2,3$ and 4 show the results of the measurements by the various liquids. An assembly of scoured wool did not sink in distilled water of any 
temperature, as Table 1 shows. This fact indicates that the penetration of a scoured wool assembly by pure water is extremely slight, no matter what degree of porosity is.

As for a greasy wool assembly dipped in distilled water, the time needed for the assembly to begin sinking varied considerably with the temperature of the water, as Table 2 shows. The reason for the considerably rapid sinking of the greasy wool assembly in high-temperature distilled water was the melting of yolk.

A scoured wool assembly sank almost in an instant in trichlene and methyl alcohol for each de-

Table 1 Time needed for scoured wool assembly to begin sinking in distilled water (where the percentage of remaining grease is $0.67 \%$ and the weight of the sinker is $7.2 \mathrm{~g}$ )

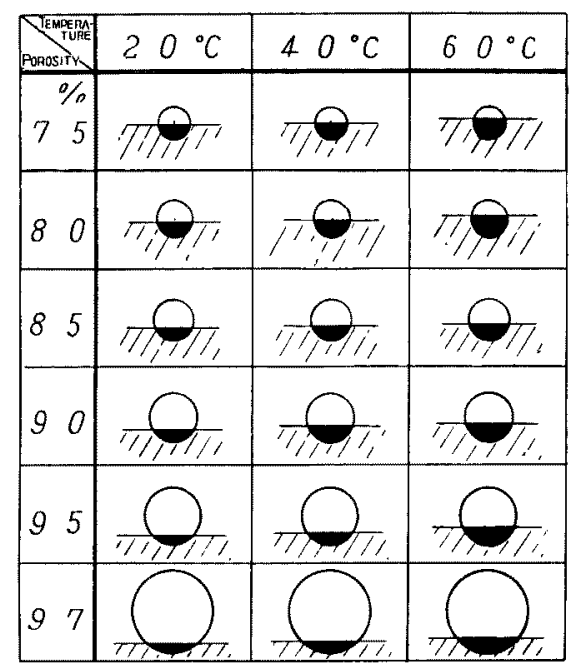

Note: The wool assembly does not sink with any combination of temperature and porosity in $10 \mathrm{hrs}$. This figure shows the cross-section of sample vessel after 10 hours of floating.

Table 3 Time needed for scoured wool assembly to begin sinking in trichlene-specific gravity 1.46 and surface tension 30.0 dyne $/ \mathrm{cm}$-where the percentage of remaining grease is $0.67 \%$, the weight of the sinker added $7.2 \mathrm{~g}$ and the temperature of liquid $20^{\circ} \mathrm{C}$.

\begin{tabular}{llc}
\hline Porosity & Time needed for wool assembly to begin sinking \\
\hline $75 \%$ & The wool assembly sinks instantly & $(0.5 \mathrm{sec})$ \\
$80 \%$ & $" 1.0 ")$ \\
$85 \%$ & $" 1.3 ")$ \\
$90 \%$ & $" 1.3 ")$ \\
$95 \%$ & $" 1.7 ")$ \\
$97 \%$ & $" 3.0$ & $(1)$ \\
\hline
\end{tabular}

gree of porosity, as Tables 3 and 4 show. It was found that the time needed for a scoured wool assembly to begin sinking in these liquids was almost the same for all degrees of porosity.

\section{Estimating the Limit of Porosity Needed for a Wool Assembly to Sink Instantly}

If a wool assembly having a constant-weight sinker is gradually reduced in its porosity degree, there develops a porosity degree which makes the assembly sink instantly when it is floated on the water

Table 2 Time needed for greasy wool assembly to begin sinking in distilled water where the percentage of grease content is $26.5 \%$ and the weight of the sinker is $7.2 \mathrm{~g}$.

\begin{tabular}{|c|c|c|c|}
\hline 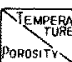 & $20^{\circ} \mathrm{C}$ & $40^{\circ} \mathrm{C}$ & $60^{\circ} \mathrm{C}$ \\
\hline 75 & & $28 \mathrm{~min}$ & $45 \mathrm{sec}$ \\
\hline 80 & & 52 & 52 \\
\hline 85 & & $1 / 3$ & 88 \\
\hline 90 & & 340 & 78 \\
\hline 95 & & & 58 \\
\hline 97 & & & 49 \\
\hline
\end{tabular}

Note: Those combination of temperature and porosity which do not make the greasy wool to sink in 10 hours are those within the solid line. The cross-sections show the state of the sample vessel after 10 hours floating.

Table 4 Time needed for scoured wool assembly to begin sinking in methyl alcohol-specific gravity 0.79 and surface tension 26.5 dyne/cm-where the percentage of remaining grease is $0.67 \%$, the weight of the sinker added $7.2 \mathrm{~g}$ and the temperature of liquid $20^{\circ} \mathrm{C}$.

\begin{tabular}{|c|c|c|c|c|c|}
\hline Porosity & Time & e needed for wo & ool assembly to & in $\sin$ & king \\
\hline $75 \%$ & The & wool assembly & sinks super-ins & $(0.2$ & sec) \\
\hline $80 \%$ & & $"$ & & $(0.3$ & \\
\hline $85 \%$ & The & wool assembly & sinks instantly & $(0.7$ & 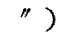 \\
\hline $90 \%$ & & $"$ & & $(0.9$ & $"$ \\
\hline $95 \%$ & & $"$ & & $(1.2$ & ") \\
\hline $97 \%$ & & $"$ & & $(2.0$ & $\pi$ \\
\hline
\end{tabular}


surface. The author calls such a porosity degree "the limit of porosity needed for the instant sinking of a wool assembly $\left({ }_{b} P_{h}\right)$."

A wool sample $W_{s}$ in weight and a sinker $W_{w}$ in weight make the total weight of the sample vessel $W_{s}+W_{w}$, which is equal to the value of the buoyancy needed to balance the combined weight of the wool sample and the vessel. Accordingly, the volume of water which should be removed to produce this buoyancy is expressible by the following formula:

$$
V_{m}=\frac{W_{s}+W_{w}}{0.998 \mathrm{~g}}
$$

where 0.998: specific gravity of water of normal temperature

The wool assembly sinks instantly if the total capacity of the sample vessel is less than the value of $V_{m n}$. The exclusive capacity of a wool assembly which has such a limit of porosity is expressible by the following formula:

$$
V_{s}=V_{m}-\frac{W_{w}}{7.86 \mathrm{~g}}
$$

where 7.86 : specific gravity of wrought steel of which vessel and sinker are made.

Accordingly, if the real volume of a wool sample is $V_{0}(5.38 \mathrm{cc})$, the value of ${ }_{l} P_{h}$ is obtainable by the following formula:

$$
{ }_{\imath} P_{h}=\frac{V_{s}-V_{0}}{V_{s}} \times 100(\%)
$$

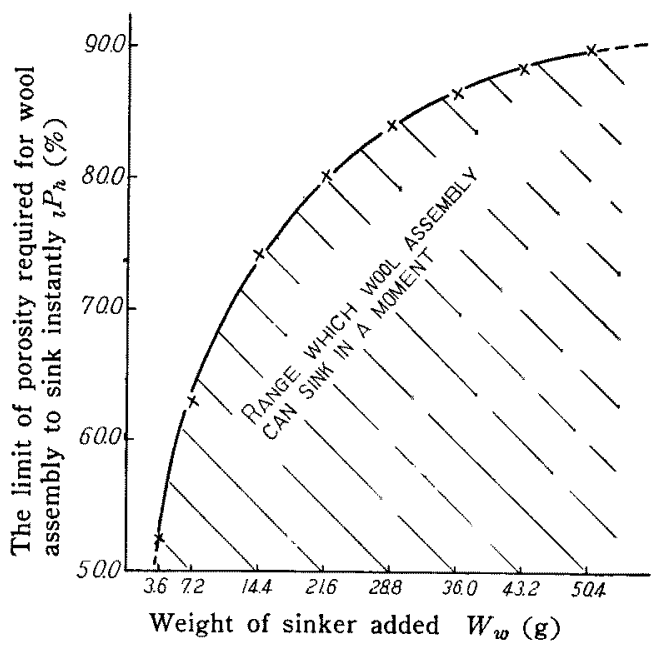

Fig. 1 Relation between limit porosity required for scoured wool assembly $7.0 \mathrm{~g}$ in absolute dry weight to sink in normaltemperature distilled water and weight of sinker required
The values of ${ }_{l} P_{h}$ obtained by equation (1) when the weight of a sample wool is $7.0 \mathrm{~g}$ is shown in Fig. 1. A heavy sinker is needed for a wool assembly of a high porosity degree to sink instantly. Every combination of porosity and sinker weight shown by the oblique line in Fig. 1 lets a wool assembly sink instantly.

\section{Estimating a Wool Assembly's Void Ca- pacity Which must Be Filled by Liquid for the Aseembly to Sink}

When a wool assembly with a sinker of a given weight is floated on a liquid surface, it is possible to estimate approximately the ratio by which the void capacity of the assembly which must be filled with a liquid to make the assembly to begin to sink.

Fig. 2 (a) shows the state of a wool assembly the instant it is floated on a liquid surface. Fig. 2(b) shows a slight penetration of the submerged section of the assembly by a liquid and a consequent slight descent of the sample vessel. The capacity $V_{m}^{\prime}$ of that part of the submerged section of the wool assembly which is the cause of buoyancy in this case is given approximately by the following formula:

$$
V^{\prime}{ }_{m} \doteqdot \frac{W_{s}+W_{w}}{\rho_{s} \cdot \mathrm{g}}
$$

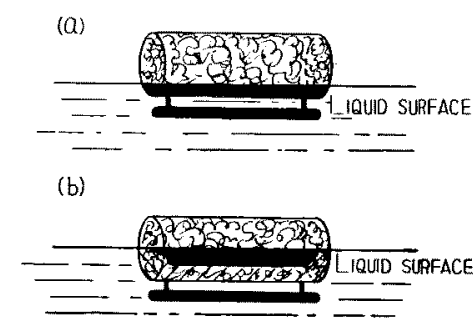

(C)

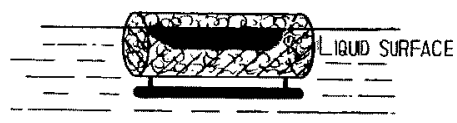

(d)

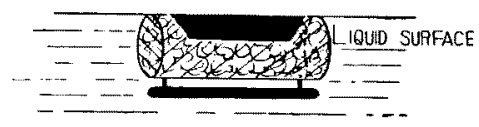

Fig. 2 The sequence in which the wool assembly is penetrated by the liquid and sinks finally (shaded part shows the part which is below liquid surface but not get penetra. ted by the liquid) 
where $\rho_{s}$ : specific gravity of liquid

If the capacity $V^{\prime}$ of the sample wool assembly alone is divided into two parts, the following equation is obtained:

$$
V_{s}^{\prime}=V_{f}+V_{p}
$$

where $V_{f}$ : the capacity of floating section

$V_{y}$ : the capacity of section submerged under liquid surface

Fig. 2 (c) shows a condition in which a liquid penetrates further into a wool assembly, thereby gradually reducing the value of $V_{f}$ and gradually increasing the velue of $V_{p}$. Dividing $V_{p}$ into two parts gives us the following equation:

$$
V_{p}=V_{p 1}+V_{p 2}
$$

where $V_{p 1}$ : the void capacity of the penetrated section

$V_{p 2}:$ the sum of the capacity of unpenetrated section and the real volume of wool fibers in the penetrated section

$V_{p 2}$ can be given by the following equation also:

$$
V_{p 2} \fallingdotseq V^{\prime}{ }_{m}-\frac{W_{w}}{7.86 \cdot \mathrm{g}}=\frac{W_{s}+W_{w}}{\rho_{8} \cdot \mathrm{g}}-\frac{W_{w}}{7.86 \cdot \mathrm{g}} \cdots
$$

Fig. 2 (d) shows a half-floating state in which the whole of a sample vessel has submerged under the liquid surface. If the liquid penetrates the wool

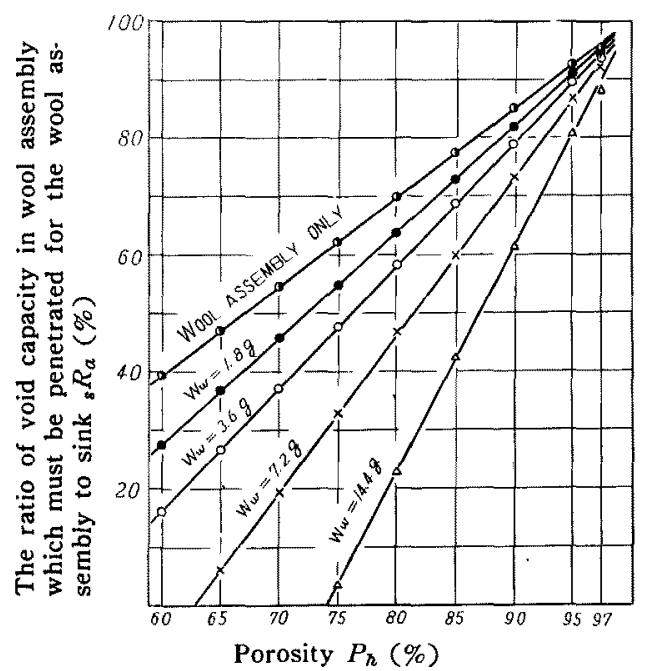

Fig. 3 Estimated values of the ratio of that void capacity in wool assembly $7.0 \mathrm{~g}$ in dry weight which must be penetrated for the wool assembly to sink in distilled water of normal temperature. $W_{w}$ shows the weights of sinkers added. assembly further and the value of $V_{p 2}$ falls below a certain value, the total weight of the sample vessel exceeds buoyancy and the wool assembly sinks. After all, the ratio ${ }_{s} R_{a}$ of the void capacity of the wool assembly which must be penetrated in order for the wool assembly to sink is obtainable by the following formula:

$$
{ }_{8} R_{a}=\frac{V_{p 1}}{V_{s}^{\prime}}=\frac{V_{s}^{\prime}-V_{p 2}}{V_{s}^{\prime}} \times 100(\%)
$$

Fig. 3 is an example of how the relation be. tween $P_{h}$ and ${ }_{8} R_{a}$ in scoured wool and in distilled water of a normal temperature is established by equation (3). The relation between $P_{h}$ and ${ }_{8} R_{a}$ is a straight line. The value of $P_{h}$, obtained as a point of intersection of this straight line and the horizontal axis, corresponds to the value of ${ }_{l} P_{h}$.

\section{Effects of Wetting Agent}

A non-ionic surface active agent of the other type was used as the wetting agent in our experiment. The concentration $C_{p}$ of the wetting agent

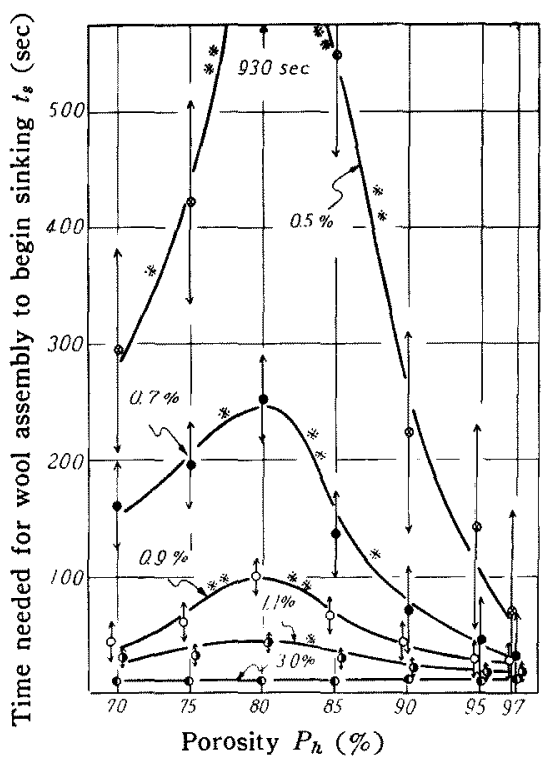

Fig. 4 Time needed for scoured wool assembly $7.0 \mathrm{~g}$ in absolute dry weight to begin sinking in non-ionic wetting agent solution of various concentration (the weight of the sinker added is $7.2 \mathrm{~g}$ and the temperature of liquid is $20^{\circ} \mathrm{C}$ ) Arrows shows confidence intervals at $5 \%$ level. *Significant difference, within $5 \%$, between two adjacent curves. * Significant difference, within $1 \%$, between two adjacent curves. 
was $0.1,0.3,0.5,0.7,0.9,1.1$ and $3.0 \%$. The sample used was scoured wool.

The wool assembly hardly sank when the wetting agent concentration $C_{p}$ was 0.1 or $0.3 \%$. Fig. 4 shows the results of the meaurements for various concentrations $C_{p}$ above $C_{p}=0.5 \%$. The time $t_{s}$ needed for sinking varies extremely widely with the concentration $C_{p}$ of the solution. The higher the
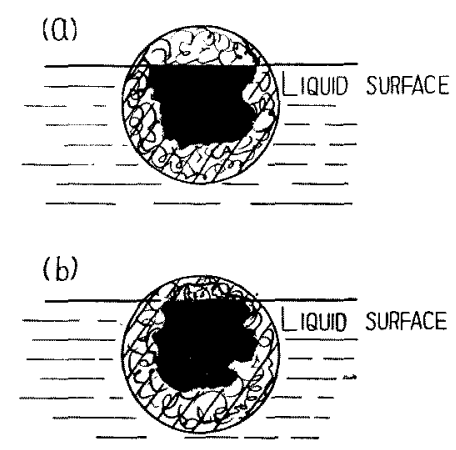

(C)

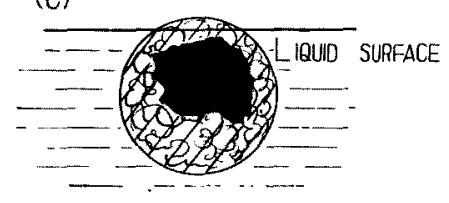

Fig. 5 Cross sections of sample vessel in half-floating state. The part penetrated by the liquid is hatched, and the part below liquid surface but not yet penetrated by the liquid is painted black

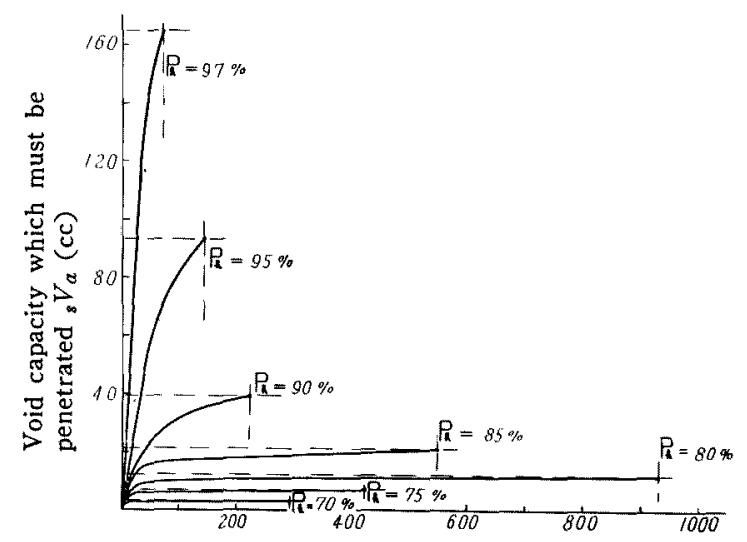

Time needed for wool assembly to begin sinking $t_{s}$ (sec)

Fig. 6 Estimated penetration of scoured wool assembly of different degree of porosity by a solution of wetting agent $0.5 \%$ in concentration and $20^{\circ} \mathrm{C}$ in temperature. The weight of the sinker added is $7.2 \mathrm{~g}$. concentration of the wetting agent, the quicker the sinking of the wool assembly.

The effect of porosity depends on whether the concentration of the wetting agent is high or low. There is no difference in $t_{8}$ between various values of porosity if the concentration of the agent is high. $t_{g}$, however, varies considerably with the degree of porosity if the concentration of the wetting agent is low, $t_{s}$ being maximum if $P_{h}=80 \%$. The more dilute the solution is, the more distinct this tendency.

The reason why $t_{s}$ is maximum if $P_{h}=80 \%$ in a dilute solution can be explained by a combination of ${ }_{8} V_{a}$ and the velocity of penetration. ${ }_{8} V_{a}$ is the void capacity which must be penetrated and which is calculable from ${ }_{s} R_{a}$ which, in turn, was obtained in the previous chapter. A wool assembly having this degree of porosity keeps up a half-floating state for a long time. A half-floating state is a condition in which a wool assembly is almost wholly submerged under the liquid surface but still does not sink (see Fig. 5).

Fig. 6 shows the curves of penetration derived from the $t_{s} \sim{ }_{s} V_{a}$ relation for $C_{p}=0.5 \%$. The velocity of penetration is almost constant if the degree of porosity is large. It falls rapidly after the first

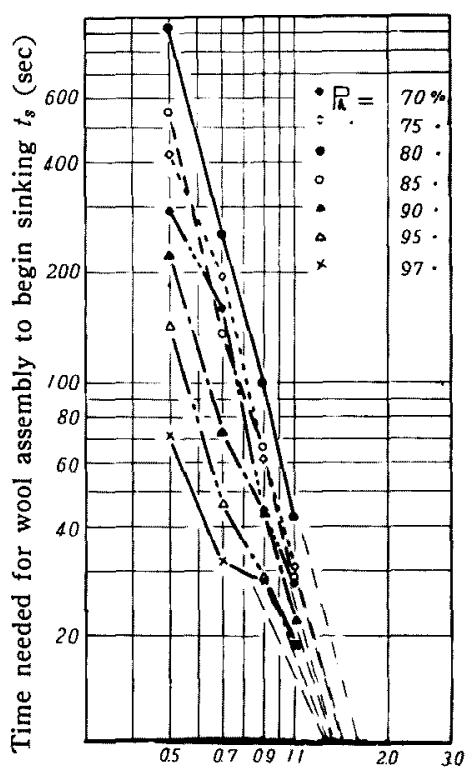

Concentration of wetting agent solution $C_{p}(\%)$

Fig. 7 The relation between the concentration of wetting agent and time needed for wool assembly to begin sinking (the absolute dry weight of scoured wool assembly is $7.0 \mathrm{~g}$, the weight of the sinker added is $7.2 \mathrm{~g}$, the temperature of liquid is $20^{\circ} \mathrm{C}$ ) 
stage of penetration, however, if the degree of porosity is small.

The relation of $\log t_{s} \sim \log C_{p}$ is shown in the log-log diagram in Fig. 7. This relation is almost a straight line when $P_{h}$ is 80 and $85 \%$, but it deviates from the straight-line relation if the degree of porosity is larger or smaller.

It may be pointed out from this fact that the degree of porosity must be fixed at a standard value to evaluate the sinking of a wool assembly experimentally. Assuming the $\log t_{s} \sim \log C_{p}$ relation to be wholly linear, this straight line was extended until it intersected $t_{s}=10$ line. The point of intersection means the concentration required of the solution in order for a wool assembly to sink in $t_{s}=10 \mathrm{sec}$. The values of concentration thus obtained are shown on the left side of Table 5 .

These values (Table 5) suggest that $C_{p}$ should be $1.68 \%$ in order for a wool assembly to sink in 10 seconds if $P_{h}$ is $80 \%$. To confirm this point, we floated wool assemblies on a solution having the concentration given in Table 5 . The time those wool assemblies took to sink in that solution is listed at the right-hand end of Table 5 . The time needed for sinking is close to $10 \mathrm{sec}$ for every degree of porosity. A rapid sinking of wool is thus made possible.

From Fig. 7 the following equation emerges as a matter of course :

$$
\log t_{s}=\log A_{p}-\alpha_{p} \log C_{p}
$$

where $A_{p}$ and $\alpha_{p}$ are constants. The rapidity of the sinking of a wool assembly when the wetting agent concentration is in the range of $C_{p}=0.5 \sim 1.5 \%$ can be compared in terms of the value of $A_{p}$.

The $P_{h} \sim A_{p}$ relation is shown in Fig. 8. As shown in Fig. 8 , the values of $P_{h}$ arranged in the order of the rapidity of sinking of scoured wool assemblies are $P_{h}=97>95>90>70>85>75>80 \%$.

Table 5 Estimated values of concentration required of wetting agent for wool assembly to sink in $10 \mathrm{sec}$. and measured values of time needed for wool assembly to begin sinking when the concentration is made equal to the estimated values

\begin{tabular}{ccc}
\hline $\begin{array}{c}\text { Porosity } \\
(\%)\end{array}$ & $\begin{array}{c}\text { Estimated values of } \\
\text { required concentration } \\
(\%)\end{array}$ & $\begin{array}{c}\text { Actually measured values } \\
\text { of time necessary for wool } \\
\text { assembly to begin sinking } \\
\text { (sec) }\end{array}$ \\
\hline 70 & 1.46 & 9.0 \\
75 & 1.55 & 9.0 \\
80 & 1.68 & 10.2 \\
85 & 1.50 & 12.2 \\
90 & 1.42 & 12.7 \\
95 & 1.39 & 11.2 \\
97 & 1.35 & 11.2 \\
\hline
\end{tabular}

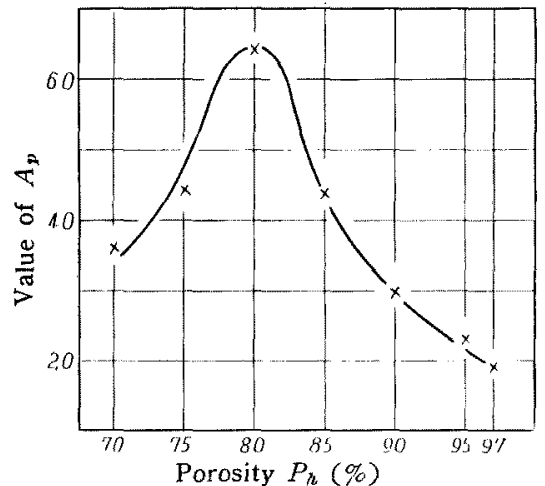

Fig. 8 Relation between $A_{p}$ and porosity $P_{r}$. A large value of $A_{p}$ indicates a slow sinking of wool assembly in a solution of wetting agent

\section{Conclusions}

To analyze the fundamental behavior of a wool assembly when it is penetrated by a treating liquid in steeping and washing bowls, the author has studied the process in a which wool assembly floating on a liquid surface begins to sink.

In actual treatment, it is desirable that a wool assembly be submerged as deep as possible in the bowl for maximum treating efficiency. Yet, in practice, a wool assembly often shows the half-floating state in the absence of a sinker. Also, it sometimes happens that a wool assembly floats on the liquid surface if the percentage of the remaining grease is small.

Judging from the results obtained from these tests, it is imperative that we use a sample fiber assembly of a constant degree of porosity in order to obtain consistent results from measuring the time required for the assembly to sink.

\section{Literature cited}

[1] H. Sanuki; J. Text. Mach. Soc. Japan, English edition, Vol. 4, No. 2, pp. 45 52 (Dec. 1958)

(2] H. Sanuki; J. Text. Mach. Soc. Japan, English edition, Vol. 5, No. 1, pp. 38 45 (June 1959)

[3] K. Ogawa; Penetrating Agent and Method of Osmosis Test, pp. 112, Maruzen, Tokyo, Japan (1942)

[4] F. M. Fuwkes: J. Phys. Cinem., Vol. 57, p. 98 (1953-1)

[5] The Society of Chemistry, Japan; Chair of Experimental Chemistry, 7-Chemistry of Surface, p. 85, Maruzen, Tokyo, Japan (1956)

[6] H. Sanuki; J. Text. Mach. Soc. Japan, English edition, Vol. 4, No. 1, pp. $17 \sim 23$ (June 1958) 